\title{
BMJ
}

\section{Longitudinal histories as predictors of future diagnoses of domestic abuse: modelling study}

\author{
Ben Y Reis, assistant professor, ${ }^{1,2}$ Isaac S Kohane, professor, ${ }^{1,2}$ Kenneth D Mandl, associate professor ${ }^{1,2}$
}

\begin{abstract}
${ }^{1}$ Children's Hospital Informatics Program at the Harvard-MIT Division of Health Sciences and Technology, Children's Hospital Boston, Boston, MA, USA
\end{abstract}

${ }^{2}$ Harvard Medical School, Boston, MA

Correspondence to: B Y Reis, 1 Autumn St, Room 540.1, Boston, MA 02115 Ben_Reis@harvard.edu

Cite this as: $B M J$ 2009;339:b3677 doi:10.1136/bmj.b3677

\begin{abstract}
Objective To determine whether longitudinal data in patients' historical records, commonly available in electronic health record systems, can be used to predict a patient's future risk of receiving a diagnosis of domestic abuse.

Design Bayesian models, known as intelligent histories, used to predict a patient's risk of receiving a future diagnosis of abuse, based on the patient's diagnostic history. Retrospective evaluation of the model's predictions using an independent testing set.
\end{abstract}

Setting A state-wide claims database covering six years of inpatient admissions to hospital, admissions for observation, and encounters in emergency departments. Population All patients aged over 18 who had at least four years between their earliest and latest visits recorded in the database (561 216 patients).

Main outcome measures Timeliness of detection, sensitivity, specificity, positive predictive values, and area under the ROC curve.

Results $1.04 \%$ (5829) of the patients met the narrow case definition for abuse, while $3.44 \%$ (19303) met the broader case definition for abuse. The model achieved sensitive, specific (area under the ROC curve of 0.88), and early (10-30 months in advance, on average) prediction of patients' future risk of receiving a diagnosis of abuse. Analysis of model parameters showed important differences between sexes in the risks associated with certain diagnoses.

Conclusions Commonly available longitudinal diagnostic data can be useful for predicting a patient's future risk of receiving a diagnosis of abuse. This modelling approach could serve as the basis for an early warning system to help doctors identify high risk patients for further screening.

\section{INTRODUCTION}

Despite the critical importance of historical data in medical decision making ${ }^{1-3}$ and the growing amount of longitudinal data available in electronic health record systems, clinicians often do not have the time or the resources to reliably access, absorb, and review all the information available to them during brief consultations..$^{4-7}$ Even with unlimited time and resources, assimilating all available information is a difficult task. Furthermore, Bodenheimer et al describe the "tyranny of the urgent" - where the brief patient-doctor visit allows time to deal with only acute situations, rather than optimise long term care. ${ }^{8}$ As a result, much of the electronic health information might not be properly interpreted, used, or even accessed, leading to potential missed diagnoses of certain clinical conditions.

One such condition is domestic abuse, ${ }^{9-11}$ which is often difficult to diagnose from a single encounter and might go unrecognised for long periods of time as it is masked by acute conditions that form the basis of clinical visits. ${ }^{11-14}$ Typically, after a diagnosis of abuse is made, a retrospective review of the longitudinal record reveals a discernable pattern of diagnoses suggestive of abuse. Domestic abuse is the most common cause of non-fatal injury to women in the United $\operatorname{States}^{9}$ and accounts for more than half the murders of women every year. ${ }^{15}$ It affects women and men and involves up to $16 \%$ of US couples a year, ${ }^{16}$ with estimates of lifetime prevalence as high as $54 \%{ }^{11}$ and lifetime risk of injury as high as $22 \% .{ }^{9}$ As undetected abuse can result in serious injury and fatality, it is critical that those at risk should be identified as early as possible. ${ }^{12} 1718$

Studies have shown that screening for domestic abuse, along with appropriate follow-up, ${ }^{14}{ }^{1920}$ can be beneficial for early detection, treatment, and prevention of future violence, and carries few if any adverse effects. ${ }^{1214172122}$ For example, one study used screening to identify 528 women as victims of intimate partner violence, of whom $443(84 \%)$ agreed to speak to an advocate, 234 (54\%) accepted case management follow-up, and $115(49 \%)$ reported that they no longer believed they were at risk of violence from their abuser three to six weeks later. ${ }^{22}$ Studies have also shown that both abused and non-abused patients favour routine screening. ${ }^{122324}$ As a result, the American Medical Association and the Joint Commission on Accreditation of Healthcare Organizations (JCAHO) have recommended routine screening for domestic abuse in the healthcare setting. ${ }^{11425}$ A recent report from the BMA (British Medical Association) urged doctors and healthcare professionals to be more vigilant for signs of domestic abuse. ${ }^{26}$ Even though some do not call for universal screening, ${ }^{27}$ many still emphasise the importance of identifying and screening high risk patients. ${ }^{28}$

Screening for domestic abuse is particularly important in the emergency department, where victims are 
Table 1| Abuse related ICD-9 codes comprising narrow case definition

\begin{tabular}{|c|c|}
\hline ICD-9 & Description \\
\hline 995.5 & Child maltreatment syndrome \\
\hline 995.50 & Child abuse, unspecified \\
\hline 995.51 & Child emotional/psychological abuse \\
\hline 995.52 & Child neglect (nutritional) \\
\hline 995.53 & Child sexual abuse \\
\hline 995.54 & Child physical abuse \\
\hline 995.59 & Child abuse/neglect (not classified elsewhere) \\
\hline 995.80 & Adult maltreatment, unspecified \\
\hline 995.81 & Adult physical abuse \\
\hline 995.82 & Adult emotional/psychological abuse \\
\hline 995.83 & Adult sexual abuse \\
\hline 995.84 & Adult neglect (nutritional) \\
\hline 995.85 & Other adult abuse and neglect \\
\hline E967.0 & $\begin{array}{l}\text { Perpetrator of child and adult abuse: by father, } \\
\text { stepfather, or boyfriend }\end{array}$ \\
\hline E967.1 & $\begin{array}{l}\text { Perpetrator of child and adult abuse: } \\
\text { by other specified person }\end{array}$ \\
\hline E967.2 & $\begin{array}{l}\text { Perpetrator of child and adult abuse: } \\
\text { by mother, stepmother, or girlfriend }\end{array}$ \\
\hline E967.3 & Perpetrator of child and adult abuse: by spouse or partner \\
\hline E967.4 & Perpetrator of child and adult abuse: by child \\
\hline E967.5 & Perpetrator of child and adult abuse: by sibling \\
\hline E967.6 & Battering by grandparent \\
\hline E967.7 & Perpetrator of child and adult abuse: by other relative \\
\hline E967.8 & $\begin{array}{l}\text { Perpetrator of child and adult abuse: } \\
\text { by non-related caregiver }\end{array}$ \\
\hline E967.9 & $\begin{array}{l}\text { Perpetrator of child and adult abuse: } \\
\text { by unspecified person }\end{array}$ \\
\hline V15.41 & History of physical abuse-rape \\
\hline V15.42 & History of emotional abuse-neglect \\
\hline V61.11 & Counselling for victim of spousal and partner abuse \\
\hline V61.21 & Counselling for victim of child abuse \\
\hline
\end{tabular}

most often encountered.$^{29} \mathrm{~A}$ three year study found that over $80 \%$ of those who experienced domestic abuse reported to the emergency department, with visits tending to peak in the month of the incident. ${ }^{30}$ The overall prevalence of domestic abuse in patients presenting to the emergency department is about $2-7.2 \%{ }^{21}$ They often present there because of limited access to traditional healthcare services, unwillingness or inability to discuss the subject with their own physician, or embarrassment or inability to present to social services outside the emergency department. ${ }^{11}$ The critical role played by emergency department clinicians in detecting domestic abuse has led to specific calls for heightened awareness for domestic abuse in presenting patients. ${ }^{1131}$

Despite the growing evidence and official recommendations, actual screening rates remain low in practice, ${ }^{162325303233}$ resulting in many missed cases of abuse, with only $5-30 \%$ of domestic abuse cases being successfully identified in the emergency department. ${ }^{163034}$ McLeer et al describe a "systems failure" in the protection of abused patients that leaves many of those passing through emergency departments unidentified and untreated.$^{35}$ In addition to low screening rates, barriers to detection include clinicians' limited encounters with the abused patients, a clinical focus on acute conditions rather than on long term issues, a lack of special training in recognising abuse, a fear of offending the patient, and a lack of resources, staff, and procedures necessary for handling abuse cases. ${ }^{11143336}$ Barriers related to the patient include their reluctance to talk, lack of awareness of provider's role, confidentiality concerns, and the attempts of patients and others to conceal abuse by offering deceptive oral histories at the time of the encounter. ${ }^{111437}$

Screening tools and scoring systems developed to assist doctors in detecting domestic abuse, ${ }^{1182138}$ whether in paper form ${ }^{21}$ or through computerised screening, ${ }^{38}$ are becoming more common. Some of these tools use clinical indicators such as the nature and anatomical site of injury, but these have limited predictive value. ${ }^{39}$ The greatest limitation of current screening approaches is that they rely on information collected from the patient during the current clinical encounter and do not take advantage of the growing amounts of longitudinal data stored in electronic health information systems.

We evaluated the usefulness of commonly available longitudinal medical information for predicting a patient's risk of receiving a future diagnosis of abuse. We developed intelligent histories-Bayesian models aimed at predicting the risk of an individual receiving a future diagnosis based on that individual's diagnostic history.

Our modelling approach could form the basis for an early warning system that monitors longitudinal health data for long term indicators of abuse risk and alerts clinicians when high risk patients are identified. As a first step towards this goal, we describe a prototype risk visualisation we are developing to provide clinicians with instant overviews of longitudinal medical histories and related risk profiles at the point of care. In conjunction with alerts for high risk patients, this could enable clinicians to rapidly review and act on all available historical information by identifying important risk factors and long term trends.

\section{METHODS}

\section{Data}

We analysed longitudinal diagnostic histories of patients aged over 18 who had at least four years between their earliest and latest diagnoses recorded in an anonymised state-wide claims database covering six years of admissions to hospital, stays at hospitals for observation, and emergency department encounters. Some 561216 patients met the inclusion criteria, having a total of 16785977 diagnoses among them.

Cases of abuse were identified according to ICD-9 (international classification of diseases, ninth revision) diagnostic codes, by using two different case definitions. The first, narrow case definition included all codes that explicitly refer to abuse (table 1 ). The second, broader case definition included the above codes, plus codes associated with intentional assault and injury (table 2). Similar case definitions based on 
Table 2|Assault and intentional injury related ICD-9 codes added to codes in table 1 to form broader case definition

\begin{tabular}{|c|c|}
\hline E960 & Fight, brawl, rape \\
\hline E960.0 & Unarmed fight or brawl \\
\hline E960.1 & Rape \\
\hline E961 & Assault-corrosive/caustic agent \\
\hline E962.0 & Assault-poisoning with medical agent \\
\hline E962.1 & Other solid and liquid substances \\
\hline E962.2 & Assault_-poisoning with gas/vapour \\
\hline E962.9 & Unspecified poisoning \\
\hline E963 & Assault-hanging/strangulation \\
\hline E964 & Assault by submersion \\
\hline E965.0 & Assault-handgun \\
\hline E965.1 & Assault-shotgun \\
\hline E965.3 & Assault-military firearms \\
\hline E965.4 & Assault-firearm (not classified elsewhere) \\
\hline E965.6 & Gasoline bomb \\
\hline E965.8 & Assault—explosive (not classified elsewhere) \\
\hline E965.9 & Unspecified explosive \\
\hline E966 & Assault by cutting and piercing instrument \\
\hline E968 & Assault by other and unspecified means \\
\hline E968.0 & Assault-fire \\
\hline E968.1 & Assault-push from high place \\
\hline E968.2 & Assault-striking with object \\
\hline E968.3 & Assault—hot liquid \\
\hline E968.4 & $\begin{array}{l}\text { Criminal neglect: abandonment of child, infant, } \\
\text { or other helpless person with intent to injure or kill }\end{array}$ \\
\hline E968.5 & Assault-transport vehicle \\
\hline E968.6 & Assault-air gun \\
\hline E968.7 & Human bite-assault \\
\hline E968.8 & Assault (not classified elsewhere) \\
\hline E968.9 & Assault (not otherwise specified) \\
\hline E969 & Late effect assault \\
\hline
\end{tabular}

ICD-9 codes have been previously validated as capturing over $95 \%$ of intentional injury cases. ${ }^{40}$

In total, 5829 patients $(1.04 \%)$ met the narrower case definition, with 511659 diagnoses among them (average of 87.8 diagnoses per patient), and 555387 patients did not meet the narrower case definition, with 16774318 diagnoses among them (average of 30.2 diagnoses per patient). Some 19303 patients (3.44\%) met the broader case definition, with 1156325 diagnoses among them (average of 59.9 diagnoses per patient), and 541913 patients did not meet the broader case definition, with 15629652 diagnoses among them (average of 28.8 diagnoses per patient).

\section{Model development}

We developed Bayesian models to estimate a patient's risk of receiving a future diagnosis of abuse based on the diagnostic history. We used naive Bayesian classifiers, ${ }^{41}$ an established modelling approach that assumes independence between the various features (diagnoses and other variables) used to classify the cases (patients) into different classes (low versus high risk of receiving a future diagnosis of abuse). Complete details of the model can be found in the technical appendix on bmj.com.
In summary, patients meeting the inclusion criteria were randomly assigned to a training set used to train the model (two thirds) or to a testing set used to validate it (one third). To account for sex specific differences in risk, we trained separate models for men and women. After training, we calculated a "partial risk score" for each diagnosis- the higher the partial risk score, the more predictive the diagnosis was of abuse. In addition to diagnoses, the model also incorporated the average number of visits a year recorded for the patient over the study period. This average number of visits, $\mathrm{v}$, was categorised into one of six groups: $v \leq 1,1<v \leq 2$, $2<\mathrm{v} \leq 4,4<\mathrm{v} \leq 6,6<\mathrm{v} \leq 10$, or $\mathrm{v}>10$, and a partial risk score was calculated for each group.

\section{Model validation}

We used the testing set, containing the remaining third of the patients, to validate the model. The model was applied retrospectively to the diagnostic histories of each patient in the testing set, analysing the data for each patient one visit at a time in chronological order and generating an "overall risk score" for the patient at the time of each new visit based on the sum of all the partial risk scores for that patient. These overall risk scores were interpreted with empirical thresholds determined according to desired specificity levels, and the corresponding sensitivity and timeliness levels were measured. To systematically gauge the actual trade-off between different levels of sensitivity and specificity in the testing set, the thresholds were set with the testing set. In an operational setting, users can set thresholds in advance based on the training set. In such a case, differences between the testing and training data might lead to a difference between desired specificity levels and actual specificity levels achieved.

\section{RESULTS}

\section{Model performance}

In predicting the risk of patients receiving future abuse diagnoses, the intelligent history models achieved an area under the ROC curve of 0.88 for the narrower case definition and 0.82 for the broader case definition.

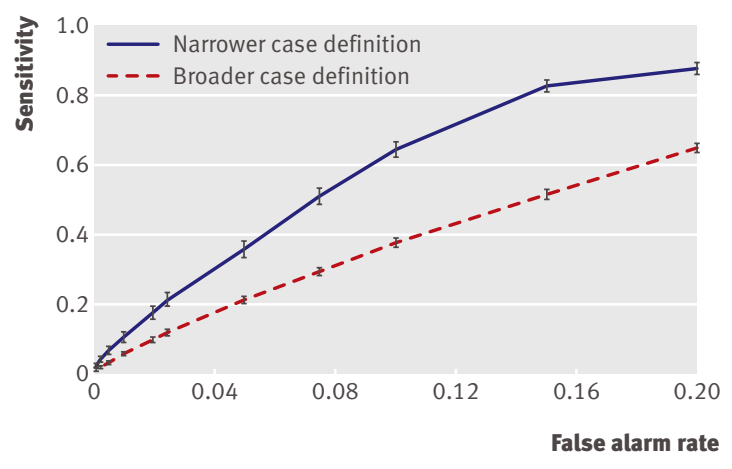

Fig 1 | Truncated ROC curve showing sensitivity (with 95\% confidence intervals) achieved by model at different benchmark false alarm rates (1-specificity). Model achieves higher sensitivity with narrower case definition (see table 1) than with broader case definition (see table 2) 
Table 3|Performance of intelligent histories models using narrower case definition of abuse and broader case definition of abuse, assault, or intentional injury

\begin{tabular}{|c|c|c|c|}
\hline $\begin{array}{l}\text { Sensitivity } \\
\text { (\%) }\end{array}$ & $\begin{array}{l}\text { Specificity } \\
(\%)\end{array}$ & $\begin{array}{r}\text { PPV } \\
(\%)\end{array}$ & $\begin{array}{l}\text { Mean days from detection } \\
\text { to first abuse diagnosis }\end{array}$ \\
\hline \multicolumn{4}{|c|}{ Narrow case definition } \\
\hline 1.8 & 99.9 & 14.4 & 280 \\
\hline 3.5 & 99.8 & 14.3 & 331 \\
\hline 3.9 & 99.75 & 13.0 & 350 \\
\hline 6.5 & 99.5 & 10.9 & 390 \\
\hline 10.3 & 99.0 & 8.9 & 459 \\
\hline 17.5 & 98.0 & 7.6 & 501 \\
\hline 21.1 & 97.5 & 7.4 & 523 \\
\hline 35.5 & 95.0 & 6.3 & 613 \\
\hline 50.8 & 92.5 & 6.0 & 661 \\
\hline 64.2 & 90.0 & 5.7 & 749 \\
\hline 82.6 & 85.0 & 4.9 & 890 \\
\hline 87.3 & 80.0 & 4.0 & 898 \\
\hline \multicolumn{4}{|c|}{ Broad case definition } \\
\hline 0.7 & 99.9 & 18.9 & 382 \\
\hline 1.4 & 99.8 & 18.6 & 364 \\
\hline 1.7 & 99.75 & 17.6 & 398 \\
\hline 2.8 & 99.5 & 15.0 & 421 \\
\hline 5.5 & 99.0 & 14.8 & 435 \\
\hline 9.6 & 98.0 & 13.0 & 501 \\
\hline 11.5 & 97.5 & 12.6 & 509 \\
\hline 20.9 & 95.0 & 11.6 & 564 \\
\hline 29.2 & 92.5 & 10.9 & 585 \\
\hline 37.3 & 90.0 & 10.5 & 620 \\
\hline 51.2 & 85.0 & 9.7 & 696 \\
\hline 64.7 & 80.0 & 9.2 & 775 \\
\hline
\end{tabular}

$\mathrm{PPV}=$ positive predictive value.

Figure 1 shows the sensitivity versus the false alarm rate. Table 3 shows the performance achieved by the models at different benchmark specificities with the narrower and broader case definitions. As expected, the relatively low prevalence of the abuse diagnosis as a percentage of all patients in the dataset resulted in a low positive predictive value, depending on the chosen level of specificity. The positive predictive value was higher for the broader case definition, where cases were relatively more common.

The model could detect high levels of risk of abuse far in advance of the first diagnosis of abuse recorded in the system (fig 2). The model detected risk of abuse an average of 10-30 months in advance, depending on the chosen level of specificity.

\section{Model composition}

Examination of the internal parameters of the model showed interesting findings. Firstly, we examined the effects of frequency of visits. As described above, each range of average number of visits a year was assigned a partial risk score. Figure 3 shows that partial risk score rises with the average number of visits a year. An increase in the number of visits would therefore increase a patient's overall abuse score. The effect seems slightly stronger (steeper slope) among women than among men.
Next, we examined the risks associated with different categories of illness. Figure 4 shows the distribution of partial risk scores in each of 12 general clinical categories. (For visualisation purposes, the diagnoses were grouped into 12 general clinical categories, based on the clinical classification software $(\mathrm{CCS})^{42}$ published by the Agency for Healthcare Research and Quality (see table A on bmj.com). These categories were used for visualisation. For modelling, each ICD-9 code was treated individually.) The category related to psychological and mental health had the highest average risk score distribution overall, followed by the injury category.

We also examined sex based differences in risk profiles. Figure 5 shows a "treemap" ${ }^{43}$ visualisation of the model for women and men. (Again, for purposes of visualisation, individual ICD-9 codes were grouped into CCS-level 2 diagnostic categories. ${ }^{42}$ ) The size of the rectangle for each diagnostic category indicates the prevalence in the abused population. The colour of each region indicates a continuous range of associated partial risk scores (from white $=$ lowest to dark red $=$ highest) for the category as a whole. Several interesting trends became evident when we compared the risks for certain diagnostic categories between the two sexes (table 4). While more abused men have alcohol related disorders, alcohol related disorders are more predictive of abuse in women than they are in men. Similarly, poisoning and injuries due to external causes are more predictive of abuse in women than they are in men. On the other hand, affective disorders, psychoses, and other mental conditions are more predictive of abuse in men than they are in women.

\section{Prototype visualisation}

We also took the first steps towards describing how these models might form the basis of an early warning system to help doctors identify high risk patients for further screening. Figure 6 shows two sample

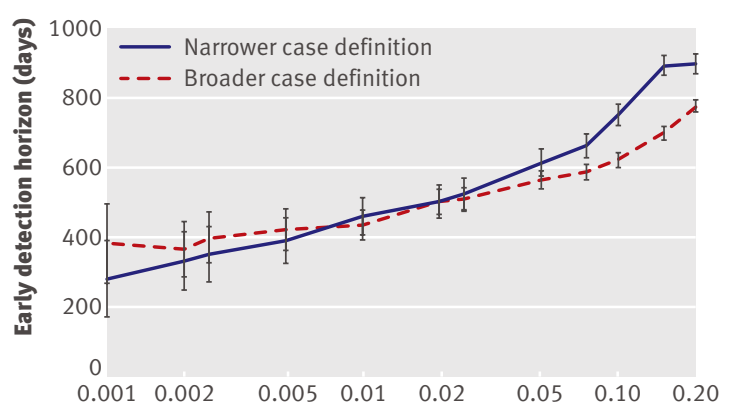

False alarm rate

Fig 2 Average time in days (with $95 \%$ confidence intervals) from initial detection of high risk of abuse to first diagnosis of abuse recorded in dataset, measured for both narrow and broad case definitions. Plot includes detected abuse cases only. Model detects risk an average of 10-30 months in advance of first recorded diagnosis, depending on desired levels of specificity (shown on log scale for clarity). At high levels of specificity, fewer cases are detected, resulting in larger confidence intervals 


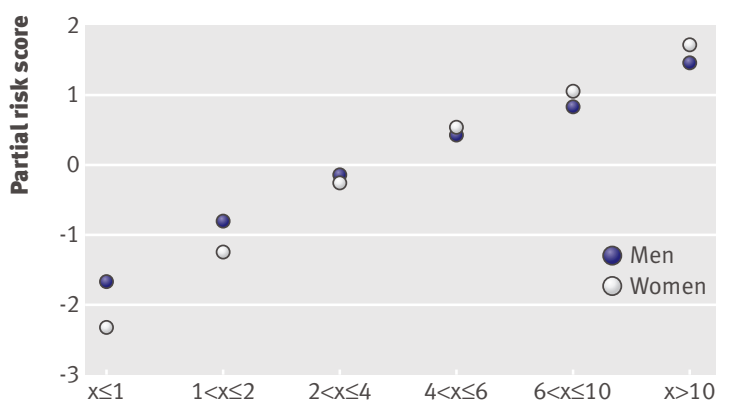

Average number of visits per year $(x)$

Fig 3 | Risk of abuse associated with average number of visits/ year. Increased risk is associated with higher average number of visits. Partial risk score is that associated with different ranges of average number of visits/year

visualisations of individual patients' histories designed to allow rapid interpretation by a clinician. Each bar represents a diagnosis, with time proceeding chronologically from the top to the bottom along the y axis. Each graph begins with the first encounter recorded for the patient (top) and ends with the first recorded diagnosis of abuse (bottom). The diagnoses are grouped into the 12 general clinical categories described above. ${ }^{42}$ The bars also represent the partial risk score assigned by the model to the particular diagnosis. For the patient in the top panel, a high risk of abuse would have been detected 27 months before the first diagnosis of abuse was recorded, given a target specificity of $95 \%$. For the patient in the lower panel, this lead time would have been 34 months.

\section{DISCUSSION}

\section{Principal findings and interpretation}

Longitudinal diagnostic data commonly available in electronic health information systems can be valuable for predicting a patient's risk of receiving a future diagnosis of abuse. Unlike previous approaches to estimating risk, ${ }^{11} 182138$ our approach examines longitudinal information rather than focusing exclusively on information collected during the present visit.

We found significant differences in longitudinal patterns of diagnoses between abused and non-abused individuals, and these differences can be used for early identification - up to years in advance - of individuals at high risk for receiving a future diagnosis of abuse. Certain broad categories of diagnoses, like psychological related conditions, were highly associated with risk of abuse. This is noteworthy as screening rates in practice have actually been found to be lower among patients presenting with psychological conditions compared with other conditions. ${ }^{32}$

Risk characteristics of specific diagnoses varied across sexes, and it is therefore useful to construct separate sex specific models of abuse risk. Abused patients had a higher average number of visits a year, ${ }^{15}$ and that this metric can be useful for differentiating between high and low risk patients.

\section{Strengths and limitations of the study}

We used a state-wide dataset covering six years of admissions to hospital, observation stays in hospital, and encounters in emergency departments. Any visits taking place outside this state, beyond this time period, or in a different care setting were not included. As a result, certain diagnoses that would have helped or hindered in identifying high risk patients might not be recorded in the dataset, thus affecting the results for that patient. Furthermore, certain people might have received a diagnosis of abuse that was not recorded in the dataset, and these people might have been misclassified as not meeting the case definition or as meeting the case definition at a different time than they actually did. Our dataset did include comprehensive coverage of all encounters in emergency departments in the state. As described above, the emergency department is where abused patients are most often encountered, ${ }^{2930}$ and such encounters are considered most critical for detecting abuse. ${ }^{1131}$ Thus we consider there is sufficient coverage for a reasonable analysis to take place.

Our case definition includes codes highly specific for abuse, assault, and intentional injury. As with all real world data, however, some visits might have been miscoded. Such omissions and inaccuracies in the data might reduce the performance of the model, but the demonstration of the utility of this approach using real world data has the potential to catalyse additional efforts in generating accurate diagnostic coding for each care episode.

Depending on the case definition used and the desired levels of specificity, the model can yield low to moderate positive predictive values (up to $14.4 \%$ for the narrow case definition and $18.9 \%$ for the broader case definition, see table 3 ). This is to be expected with conditions having a low prevalence (in the present case, $1.04 \%$ with the narrow definition and $3.44 \%$ with the broad definition), as the positive

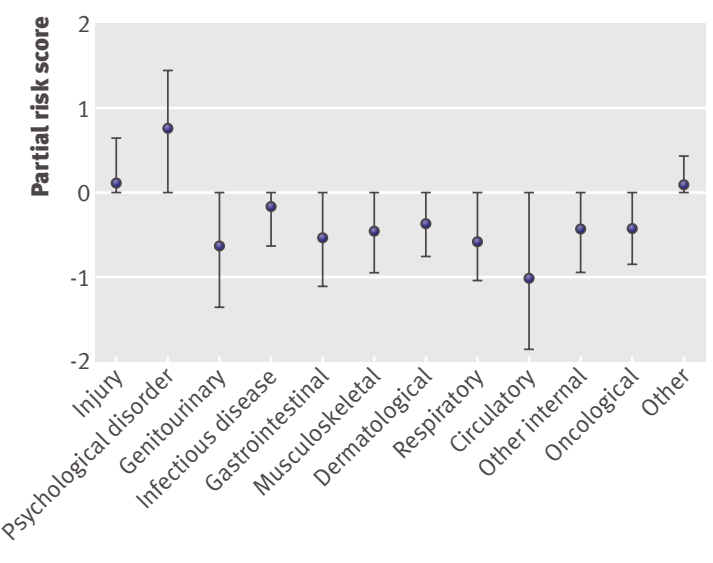

Fig 4 | Distribution of abuse risk by general clinical category (see table A on bmj.com). Means and $25 \%$ and $75 \%$ centiles shown for each category. On average, diagnostic categories related to injury and psychological health are most predictive of abuse 


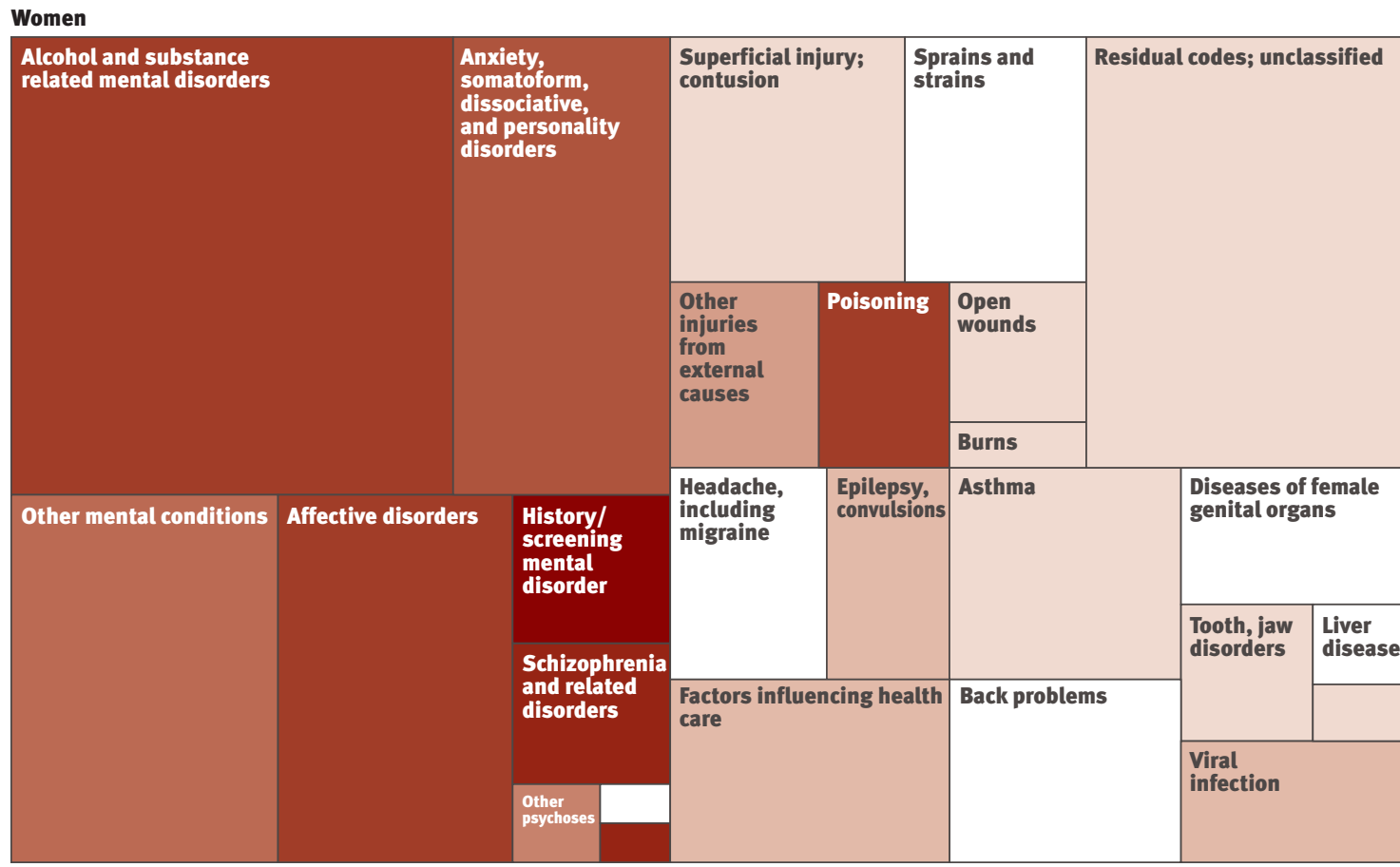

Men

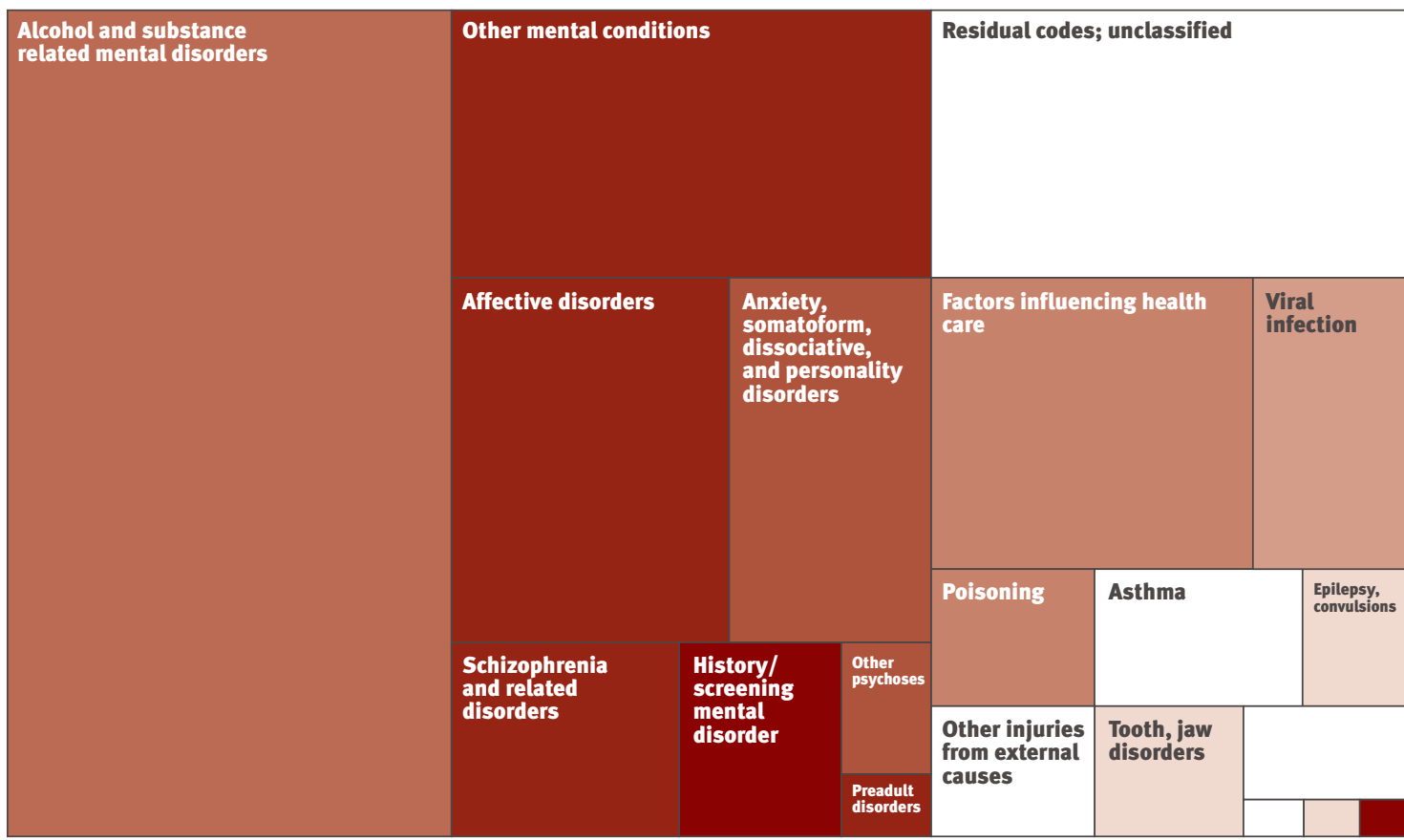
More predictive

Fig 5| "Treemap" visualisations of abuse risk associated with different diagnostic categories for women and men. Each rectangle represents a different clinical diagnostic category. Area of rectangle indicates prevalence of that category in abused population (only most prevalent conditions are shown). Colour indicates how predictive that diagnostic category is of receiving a future abuse diagnosis (white $=$ lowest, dark red $=$ highest). For example, alcohol and substance related disorders, the most prevalent category in men and women, are more prevalent among abused men than abused women (larger rectangle) but more predictive of abuse in women than men (darker red colour). Comparison between men and women in table 4, shows additional differences 
Table $4 \mid$ Partial risk scores* for women and men for select clinical categories

\begin{tabular}{llc} 
Category $\dagger$ & Women $(95 \% \mathrm{Cl})$ & Men (95\% Cl) \\
Alcohol, substance related mental disorders & $1.455(1.440$ to 1.471$)$ & $1.253(1.235$ to 1.271$)$ \\
\hline Injuries from external causes & $0.885(0.843$ to 0.925$)$ & $0.175(0.098$ to 0.249$)$ \\
\hline Poisoning & $1.326(1.279$ to 1.373$)$ & $1.039(0.960$ to 1.115$)$ \\
\hline Affective disorders & $1.435(1.410$ to 1.459$)$ & $1.726(1.688$ to 1.764$)$ \\
\hline Other mental conditions & $1.283(1.260$ to 1.305$)$ & $1.640(1.606$ to 1.673$)$ \\
\hline Other psychoses & $1.065(0.980$ to 1.148$)$ & $1.326(1.209$ to 1.434$)$ \\
\hline
\end{tabular}

*The higher the partial risk score, the more predictive the category of diagnoses is of abuse.

fFirst three categories listed are more predictive of abuse in women than in men. Second three categories listed are more predictive of abuse in men than in women.

predictive value is directly proportional to prevalence of the condition being detected. These levels could be clinically useful in settings where the model is being used to identify patients for whom standard screening should be performed, especially when screening rates in practice remain below desired levels. ${ }^{162325303233}$

We focused on predicting the risk of future diagnoses of abuse, and the model is trained on patients who have been diagnosed in a clinical setting. Potential differences between cases of abuse that typically get diagnosed versus those cases that typically do not get diagnosed might serve as an important bias and might hinder the model's ability to detect the latter. As mentioned above, however, domestic abuse often goes undiagnosed or is diagnosed only after considerable delay. Given the current high levels of underdiagnosis, it is likely that use of the model in a clinical setting would lead to the detection of some of the cases that are currently not typically diagnosed. The effect of implementing such a model in clinical practice is an important empirical question for future research.

Differences in care and coding practices might affect the generalisability of models from one health environment to another. We therefore recommend the training of a specific model for each healthcare environment. We expect the modelling approach to be generalisable to other settings inside and outside the US, as the minimal set of data elements (ICD-9 codes, dates of visits) used by the model are commonly stored throughout many countries with electronic medical record systems or claims systems. In countries that do not yet have electronic medical record systems, these models would be difficult to implement, though with time, electronic medical record systems are being deployed more widely throughout the world.

Our goal was to predict a patient's risk of receiving a future diagnosis of abuse, based on the patient's longitudinal diagnostic record to date. This prediction can help care givers to identify individuals who fall into either of two categories: those who may be currently experiencing abuse but have yet to be diagnosed and those who are not yet experiencing abuse but are at a high risk of being abused in the future. Currently, the model does not differentiate between these two types, though this is an important area for future research, as such a differentiation might enable explicit attempts to estimate time to event.

\section{Future research}

Further aspects are worthy of future study. Currently, the risk associated with each diagnosis is modelled separately. More complex models can be developed to explicitly incorporate the relations between multiple diagnostic codes - for example, the presence of diagnosis A together with diagnosis B might be more or less predictive of abuse risk than the combination of the individual risks of $\mathrm{A}$ or $\mathrm{B}$ alone.

While the present analysis relied on claims data, the structured information and text available in more comprehensive electronic health information systems can provide a richer substrate for future intelligent history models. Explicitly modelling temporality, such as the order in which visits occurred and the intervals of time between certain diagnoses, might further improve performance.

With proper integration into the clinical workflow, the intelligent history could aid the already overloaded clinician in identifying high risk patients who warrant further in-depth screening by the clinician. Such screening must always take place in the context of proper training for physicians in handling abuse and an environment that offers appropriate resources and referrals for abused patients. ${ }^{14} 1920$ It is important to emphasise that an early warning system based on intelligent history models would not be intended for making the diagnosis of abuse but rather for identifying patients who are at high risk of receiving a future abuse diagnosis and therefore warrant screening. This is especially important in settings where screening rates in practice remain below desired levels. ${ }^{162325303233}$

Potential next steps towards the development of an early warning system for clinicians would include automation of the intelligent history as a service-oriented tool, and rigorous design work on the human interface to refine and test the numerical and visual presentation in creating an early warning system for clinicians. The approach would work as follows. A patient's longitudinal medical history accumulates over time inside an electronic health record system. Whenever new information is recorded for the patient, the intelligent histories model re-analyses the information accumulated to date to estimate the patient's risk of receiving a future diagnosis of abuse. The patient's physician is notified if the patient is at high risk of abuse. The physician uses the visualisation to quickly review the patient's past diagnoses and identify important long term trends in the patient's history. The risk estimate, together with the high level view of the patient's diagnostic history, enables the physician to make a better informed decision about whether to proceed with further screening of the patient. In this way, the intelligent histories model could improve screening by helping physicians to identify high risk patients who might otherwise be missed.

In conclusion, our findings suggest that the vast quantities of longitudinal data accumulating in 


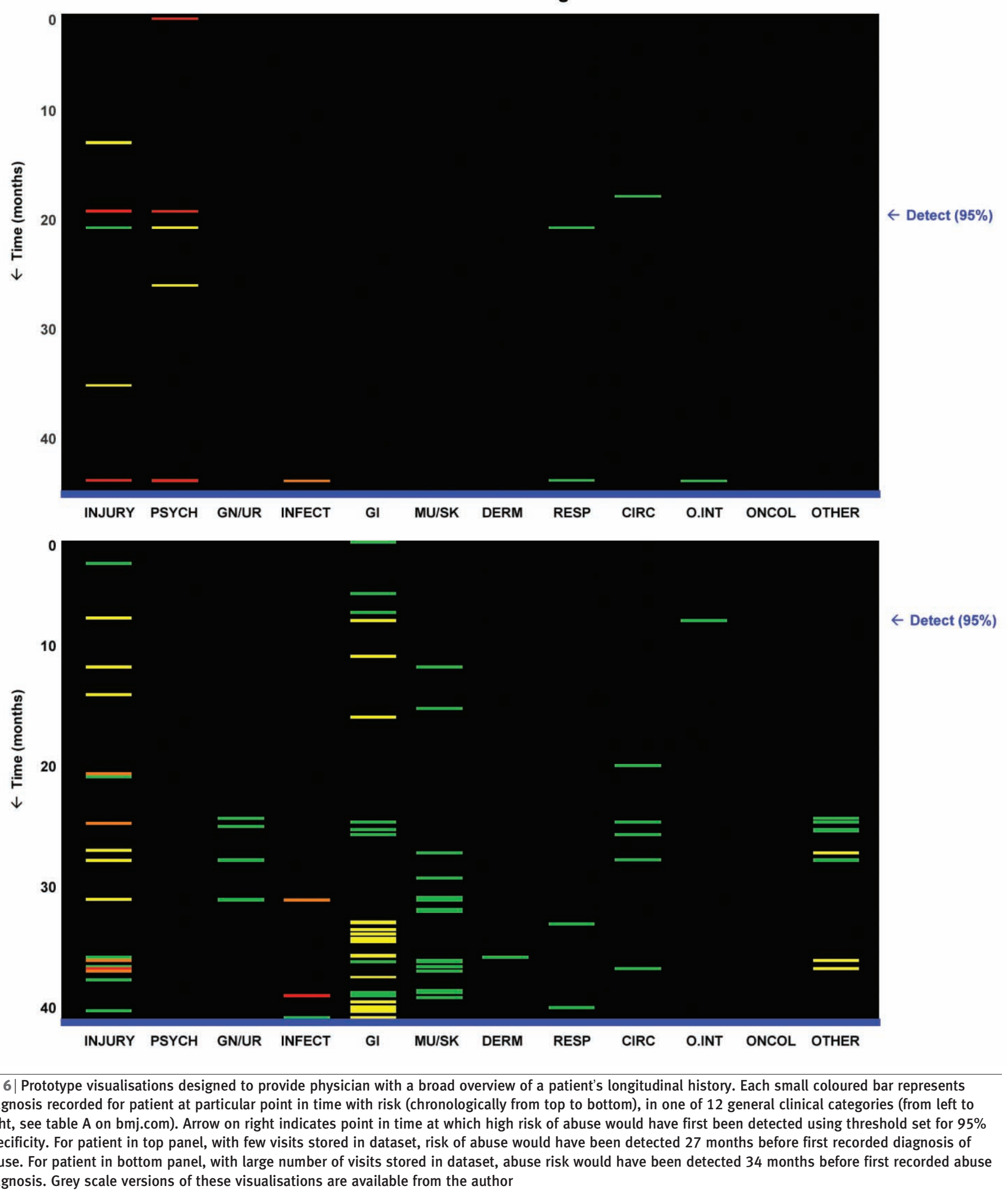

Fig 6 | Prototype visualisations designed to provide physician with a broad overview of a patient's longitudinal history. Each small coloured bar represents diagnosis recorded for patient at particular point in time with risk (chronologically from top to bottom), in one of 12 general clinical categories (from left to right, see table A on bmj.com). Arrow on right indicates point in time at which high risk of abuse would have first been detected using threshold set for $95 \%$ specificity. For patient in top panel, with few visits stored in dataset, risk of abuse would have been detected 27 months before first recorded diagnosis of diagnosis. Grey scale versions of these visualisations are available from the author

electronic health information systems present an untapped opportunity for improving medical screening and diagnosis. In addition to the direct implications for prediction of risk of abuse, the general modelling framework presented here has far reaching potential implications for automated screening of other clinical conditions where longitudinal historical information can be useful for estimating clinical risk. 


\section{WHAT IS ALREADY KNOWN ON THIS TOPIC}

Domestic violence is a dangerous condition that is difficult to detect, and screening rates are low

Diagnostic histories might be useful in identifying patients who are at high risk of abuse, but physicians typically do not have time to thoroughly review this information during the course of a clinical visit

\section{WHAT THIS STUDY ADDS}

Longitudinal medical information commonly available in electronic health systems can be useful for predicting the risk of a patient receiving a future diagnosis of abuse

The Bayesian models used can serve as the basis for a future early warning system that could help doctors to identify high risk patients for further screening

We thank Karen Olson for preparing the dataset for analysis. Contributors: BYR designed the study, developed the models, analysed the results, wrote the manuscript, and is guarantor. ISK contributed to study design and writing the manuscript and advised on clinical issues. KDM contributed to study design and writing the manuscript and advised on clinical issues.

Funding: This work was supported by the US Centers for Disease Control and Prevention (grant R01 PH000040) and the National Library of Medicine (grants R01 LM009879, R01 LM007677, and G08LM009778). The funders have no involvement with the research.

Statement of independence of researchers from funders: The authors and the research are completely independent of the funders.

Competing interests: None declared.

Ethical approval: This study was approved by the institutional review board approval.

1 Coleman EA. Falling through the cracks: challenges and opportunities for improving transitional care for persons with continuous complex care needs. J Am Geriatr Soc 2003;51:549-55.

2 Stiell A, Forster AJ, Stiell IG, van Walraven C. Prevalence of information gaps in the emergency department and the effect on patient outcomes. CMAI 2003;169:1023-8.

3 Overhage JM, Dexter PR, Perkins SM, Cordell WH, McGoff J, $M c G r a t h ~ R$, et al. A randomized, controlled trial of clinical information shared from another institution. Ann Emerg Med 2002;39:14-23.

4 Hripcsak G, Sengupta S, Wilcox A, Green RA. Emergency departmen access to a longitudinal medical record. J Am Med Inform Assoc 2007;14:235-8.

5 Noone J, Warren J, Brittain M. Information overload: opportunities and challenges for the GP's desktop. Medinfo 1998;9:1287-91.

6 Barnett GO, Barry MJ, Robb-Nicholson C, Morgan M. Overcoming information overload: an information system for the primary care physician. Medinfo 2004;11:273-6.

7 Hall A, Walton G. Information overload within the health care system a literature review. Health Info Libr / 2004;21:102-8.

8 Bodenheimer T, Wagner EH, Grumbach K. Improving primary care for patients with chronic illness. JAMA 2002;288:1775-9.

9 Kyriacou DN, Anglin D, Taliaferro E, Stone S, Tubb T, Linden JA, et al. Risk factors for injury to women from domestic violence against women. N Engl J Med 1999;341:1892-8.

10 Saltzman LE, Green YT, Marks JS, Thacker SB. Violence against women as a public health issue: comments from the CDC. Am J Prev Med 2000;19:325-9.

11 Ernst AA, Weiss SJ. Intimate partner violence from the emergency medicine perspective. Women Health 2002;35:71-81.

12 Ross J, Walther V, Epstein I. Screening risks for intimate partner violence and primary care settings: implications for future abuse. Soc Work Health Care 2004;38:1-23.

13 McLeer SV, Anwar RA. The role of the emergency physician in the prevention of domestic violence. Ann Emerg Med 1987;16:1155-61.

14 Houry D, Kaslow NJ, Kemball RS, McNutt LA, Cerulli C, Straus H, et al. Does screening in the emergency department hurt or help victims of intimate partner violence? Ann Emerg Med 2008;51:433-42, 442 e1-7.

15 Koss MP, Koss PG, Woodruff WJ. Deleterious effects of criminal victimization on women's health and medical utilization. Arch Intern Med 1991;151:342-7.

16 Thompson RS, Rivara FP, Thompson DC, Barlow WE, Sugg NK, Maiuro RD, et al. Identification and management of domestic violence: a randomized trial. Am J Prev Med 2000;19:253-63.
17 McNulty A, Andrews P, Bonner M. Can screening for domestic violence be introduced successfully in a sexual health clinic? Sex Health 2006;3:179-82.

18 Houry D, Feldhaus K, Peery B, Abbott J, Lowenstein SR, al -Bataa-de-Montero S, et al. A positive domestic violence screen predicts future domestic violence. J Interpers Violence 2004;19:955-66.

19 Groth B, Chelmowski MK, Batson TP. Domestic violence: level of training, knowledge base and practice among Milwaukee physicians. WMJ 2001;100:24-8, 36.

20 Liebschutz J, Battaglia T, Finley E, Averbuch T. Disclosing intimate partner violence to health care clinicians-what a difference the setting makes: a qualitative study. BMC Public Health 2008;8:229.

21 Heron SL, Thompson MP, Jackson E, Kaslow NJ. Do responses to an intimate partner violence screen predict scores on a comprehensive measure of intimate partner violence in low-income black women? Ann Emerg Med 2003;42:483-91.

22 Krasnoff M, Moscati R. Domestic violence screening and referral can be effective. Ann Emerg Med 2002;40:485-92.

23 Glass N, Dearwater S, Campbell I. Intimate partner violence screening and intervention: data from eleven Pennsylvania and California community hospital emergency departments. J Emerg Nurs 2001;27:141-9.

24 Gielen AC, O'Campo PJ, Campbell JC, Schollenberger J, Woods AB, Jones AS, et al. Women's opinions about domestic violence screening and mandatory reporting. Am J Prev Med 2000;19:279-85.

25 Abbott J, Johnson R, Koziol-McLain J, Lowenstein SR. Domestic violence against women. Incidence and prevalence in an emergency department population. JAMA 1995;273:1763-7

26 Kmietowicz Z. Greater vigilance needed to tackle domestic abuse, says BMA. BMJ 2007;334:1289.

27 Ramsay J, Richardson J, Carter YH, Davidson LL, Feder G. Should health professionals screen women for domestic violence? Systematic review. BMJ 2002;325:314.

28 Goodyear-Smith F. National screening policies in general practice: a case study of routine screening for partner abuse. Appl Health Econ Health Policy 2002;1:197-209.

29 McCloskey LA, Lichter E, Ganz ML, Williams CM, Gerber MR, Sege R, et al. Intimate partner violence and patient screening across medical specialties. Acad Emerg Med 2005;12:712-22.

30 Kothari CL, Rhodes KV. Missed opportunities: emergency department visits by police-identified victims of intimate partner violence. Ann Emerg Med 2006;47:190-9.

31 Dearwater SR, Coben JH, Campbell JC, Nah G, Glass N, McLoughlin E, et al. Prevalence of intimate partner abuse in women treated at community hospital emergency departments. JAMA 1998;280:433-8.

32 Larkin GL, Hyman KB, Mathias SR, D'Amico F, MacLeod BA. Universal screening for intimate partner violence in the emergency department: importance of patient and provider factors. Ann Emerg Med 1999;33:669-75.

33 Waalen J, Goodwin MM, Spitz AM, Petersen R, Saltzman LE. Screening for intimate partner violence by health care providers. Barriers and interventions. Am J Prev Med 2000;19:230-7.

34 McLeer SV, Anwar R. A study of battered women presenting in an emergency department. Am J Public Health 1989;79:65-6.

35 McLeer SV, Anwar RA, Herman S, Maquiling K. Education is not enough: a systems failure in protecting battered women. Ann Emerg Med 1989;18:651-3.

36 Ortiz JJ, Ford LR. Existence of staff barriers to partner violence screening and screening practices in military prenatal settings. J Obstet Gynecol Neonatal Nurs 2005;34:63-9.

37 McCloskey LA, Williams CM, Lichter E, Gerber M, Ganz ML, Sege R. Abused women disclose partner interference with health care: an unrecognized form of battering. J Gen Intern Med 2007;22:1067-72.

38 Trautman DE, McCarthy ML, Miller N, Campbell JC, Kelen GD. Intimate partner violence and emergency department screening: computerized screening versus usual care. Ann Emerg Med 2007;49:526-34.

39 Fanslow JL, Norton RN, Spinola CG. Indicators of assault-related injuries among women presenting to the emergency department. Ann Emerg Med 1998;32:341-8.

40 Stone DA, Kharasch SJ, Perron C, Wilson K, Jacklin B, Sege RD. Comparing pediatric intentional injury surveillance data with data from publicly available sources: consequences for a public health response to violence. Inj Prev 1999;5:136-41.

41 Kononenko I. Machine learning for medical diagnosis: history, state of the art and perspective. Artif Intell Med 2001;23:89-109.

42 HCUP CCS. Healthcare cost and utilization project (HCUP). August 2009. Rockville, MD: Agency for Healthcare Research and Quality. www.hcup-us.ahrq.gov/toolssoftware/ccs/ccs.jsp.

43 Bederson BB, Shneiderman B, Wattenberg M. Ordered and quantum treemaps: making effective use of 2D space to display hierarchies. ACM Trans Graph 2002;21:833-54.

Accepted: 26 May 2009 